Vol 11, Issue 12, 2018

\title{
IMMUNOHISTOCHEMICAL EXPRESSION OF THE PROMISING THERAPEUTIC TARGET (HUMAN EPIDERMAL GROWTH FACTOR RECEPTOR 2/NEU) IN IRAQI PATIENTS WITH MEDULLOBLASTOMA
}

\author{
ZAINAB KHALID AL MUKHTAR ${ }^{1}$, ZINAH HUSSEIN ABED ALI AL SAEG ${ }^{2}$, FARAH FALAH HASAN ${ }^{3}$ \\ ${ }^{1}$ Department of Pathology, College of Medicine, Baghdad University, Baghdad, Iraq. ${ }^{2}$ Department of Pathology, Medical City Complex, \\ Baghdad, Iraq. ${ }^{3}$ Department of Pathology, College of Medicine, Karbala University, Karbala, Iraq. Email: zainabks48@gmail.com, \\ Zinaalsayag441@gmail.com,dr.farah.histopathology@gmail.com
}

Received: 09 July 2018, Revised and Accepted: 09 August 2018

ABSTRACT

Objectives: With the advent of ongoing novel modalities toward the treatment of human epidermal growth factor receptor 2 (HER2)/NEU - positive malignancies, the serious side effects of chemoradiotherapy have been minimized. Hence, this study was conducted to identify the patterns of immunohistochemical expression of the promising therapeutic target (HER2/NEU) among Iraqi patients with medulloblastoma in an attempt to provide basic histological information's that would help in future clinical researches.

Materials and Methods: In this retrospective study, 42 formalin - fixed paraffin - embedded tissue blocks represent cases of surgically removed medulloblastomas were retrieved from the archived materials in a specialized surgical hospital at Bagdad. The histological diagnosis had been revised, and all cases were stained by the immunohistochemical technique with HER2/NEU antibody and assessed independently by three pathologists.

Results: Out of 42 cases, only two which represent $4.76 \%$ showed positive results manifested by a strong membranous staining when immunohistochemically evaluated using the same scoring system established for HER2/NEU in breast cancer. 14 cases (33.3\%) showed incomplete membranous and five cases (11.9\%) showed only cytoplasmic reaction patterns.

Conclusion: The rate of expression of HER2/NEU in medulloblastoma among Iraqi patients is very low and found only in aggressive anaplastic histological type when immunohistochemically evaluated using the same scoring system established for HER2/NEU in breast cancer. However, a good number of negative cases showed cytoplasmic and incomplete membranous staining patterns highlighting the importance of establishing medulloblastoma - specific HER2/NEU scoring criteria and testing methods to discover the unique feature of expression of this therapeutic target in medulloblastoma.

Keywords: Medulloblastoma, Human epidermal growth factor receptor 2/NEU, Immunohistochemistry.

(c) 2018 The Authors. Published by Innovare Academic Sciences Pvt Ltd. This is an open access article under the CC BY license (http://creativecommons. org/licenses/by/4. 0/) DOI: http://dx.doi.org/10.22159/ajpcr.2018.v11i12.28373

\section{INTRODUCTION}

Medulloblastoma is the most common malignant tumor of the central nervous system in children [1] in which it constitutes about $20 \%$ of all intracranial tumors and $40 \%$ of all childhood posterior fossa tumors [2], it is highly invasive pediatric brain tumor with fast cellular proliferation that commonly disseminated throughout the central nervous system at presentation [3]. According to the recent World Health Organization (WHO), medulloblastomas are grouped based on molecular features in addition to histopathological characteristics for more accurate assessment of risk in children, this molecular classification relates to the clustering of medulloblastomas into groups on the basis of transcriptome or methylome profiling [4]. The major histological subtypes of medulloblastoma are classic, desmoplastic/ nodular medulloblastoma, medulloblastoma with extensive nodularity, and large cell or anaplastic medulloblastomas, each has their own particular clinical associations [5-8], a distinct morphology, age of onset, and prognosis [9]. Irrespective of their histological or genetic features, medulloblastomas are considered as Grade IX according to (WHO) grading system which is regarded as the highest histological grade [10].

HER2/NEU oncogene is a member of the tyrosine kinase family similar to the epidermal growth factor receptor (EGFR), it is located on chromosome $17 \mathrm{q} 21$ and encodes a $185 \mathrm{kd}$ transmembrane protein that lacks a natural legend [11]. HER-2 activation starts off signal cascades including the MAPK and PI3K/AKT pathways in which they are necessary for cell proliferation and differentiation [12]. Overactivity of EGFR signaling leads to uncontrolled cellular proliferation and increased survival which leads to cellular transformation and tumor progression [11]. Medulloblastoma has been reported in the literature to have an overexpression of HER2/NEU in contrast to normal cerebellar tissue $[13,14]$. The successful story of anti-HER2/NEU (trastuzumab) in the treatment of advanced metastatic breast cancer has not only led scientists in developing newer molecules and targets against HER-2 but also has been tried as a therapeutic option in other malignancies [15]. Although HER2/NEU expression in medulloblastoma approved to be relatively lower than in breast cancer rendering monoclonal antibodies may be ineffective, a recent study suggests new modality about adoptive transfer of HER2-specific $\mathrm{T}$ cells in a promising immunotherapeutic approach for medulloblastoma [16]. To identify new targeting drug delivery systems for malignancy, cancer cell biology needs to be extensively understood [17]. Furthermore, there is an urgent need for novel therapy in very young child with medulloblastoma that improves dangerous toxicity so this study was conducted to identify the patterns of immunohistochemical expression of human epidermal growth factor receptor (HER2/NEU) in the most common pediatric intracranial malignancy (medulloblastoma) in an attempt to provide basic histological information that would help in future researches.

\section{METHODS}

In this retrospective study covering the period from January 2014 to November 2017, 42 formalin - fixed paraffin - embedded tissue blocks with initial diagnosis of medulloblastoma were collected from the archived materials in the Histopathology Department of Gazy Al Hariri 
teaching hospital for surgical specialization at Bagdad, the paraffin blocks represented all cases of surgically removed posterior fossa medulloblastomas in at that period. Clinicopathological parameters such as age, gender of patient, and histological/morphological types were obtained from the available histopathologic reports.

Two sections of $5 \mathrm{Mm}$ thickness were taken from each block; the first was stained by hematoxylin and eosin ( $\mathrm{H}$ and $\mathrm{E})$ for histopathologic revision, the other section put in a positively charged slide and stained immunohistochemical for HER2/NEU.

The $\mathrm{H}$ and $\mathrm{E}$ stained sections were reassessed for the morphological types of the tumor by three independent pathologists. For detection of HER2/ NEU antigen, we used polyclonal rabbit antihuman c-erb-2 oncoprotien (Code A0485) manufactured by DAKO with a dilution of 1:1000, sections from the breast carcinomas that known to be immunoreactive for HER2/NEU were used as a positive control, while the negative control slides were obtained by omitting the primary antibody. All sections were stained at the governmental hospital using autostainer equipment $(X$ biogenex i6000). To perform immunohistochemical staining bimanually on each block, $5 \mathrm{~mm}$ sections were made and applied the section on a positive charge dewaxing paraffin-embedded sections. After antigen retrieval was performed, the tissue sections were covered with peroxide block solution for 5 min followed by incubation of primary antibody for $30 \mathrm{~min}$, then the tissue sections covered with poly excel poly HRP and incubate for $30 \mathrm{~min}$ at room temperature, working solution and incubate for $5 \mathrm{~min}$ at room temperature, and finally hematoxyline stain was applied. Immunohistochemically stained sections were evaluated under a light microscope(leica DM500) independently by three pathologists by using the same scoring system established to evaluate HER2/NEU in breast cancer [18].

Score 0 no staining or membranous staining is observed in $<10 \%$ of the tumor cells.

Score $1+$ partial faint membrane staining in more than $10 \%$ of the tumor cells.

Score $2+$ circumferential weak to moderate membrane staining observed in more than $10 \%$ of the tumor cells.

Score $3+$ circumferential strong membrane staining observed in more than $10 \%$ of the tumor cells. Scores of $2+$ and $3+$ were considered positive for HER2/NEU.

Photomicrographs were taken in this study with a camera (Leica Icc 50E).

This retrospective study was approved by the ethical committee of the national center for educational laboratories and done in accordance with it is an institutional policy in which patient consent was taken at the beginning of consultation for any future studies.

\section{Statistical analysis}

Satistial analysis was performed with SPSS versus 25.

\section{RESULTS}

A total of 42 tissue samples representing medulloblastomas were included in this retrospective study. The mean age of patients with medulloblastoma was 7.53 years, with age range of $1-22$ years. The distribution of medulloblastoma cases according to age groups was shown in Table 1.

The present study showed that $27 / 42(64.28 \%)$ of cases were males and $15 / 42(35.71 \%)$ were females, with a male to female ratio was 1.8:1.

Regarding histological types, the present study showed that classic medulloblastoma was $31 / 42$ representing $73.80 \%$ (Fig. 1), nodular/ desmoplastic medulloblastoma was 6/42 (14.28\%), medulloblastoma with extensive nodularity was $3 / 42$ (7.14\%), and only $2 / 42(4.76 \%)$ were large cell/anaplastic medulloblastoma (Table 2).

After immunohistochemical search for HER2/NEU in 42 cases, only two cases showed positive membranous staining scored as +3 (Figs. 2 and 3) both were of anaplastic morphology. Of 40 negative cases, 5 cases showed only cytoplasmic reactivity, 2 showed positive nuclear reactivity, and 14 cases showed incomplete membranous staining (Fig. 4). According to the scoring system used in this study, only two cases (represent $4.76 \%$ ) were positive for HER2/NEU antibody.

\section{DISCUSSION}

This study is not a large epidemiological study that expresses the prevalence and incidence of different clinicopathologic features of medulloblastoma. However, in general, the overall mean age in the present study was 7.47 years, and it was relatively uncommon in adults, these findings agreed with the studies of Chan et al. and Kumar et al. [19,20] which also found that it was more common in children at their first decade of life. Regarding gender in this study a male predominance was found, with a male to female ratio 1.8:1, this results agreed with the studies of Curran et al. and Herms et al. [21,22]. Histological examination of medulloblastoma in the present study showed a clear predominance of classic morphology which was in agreement with the studies done by Kumar et al., Massimino et al., and Nam et al. [6,20,23].

Table 1: Distribution of medulloblastoma cases according to age groups of patients

\begin{tabular}{ll}
\hline Age group & Frequency (\%) \\
\hline $1-10$ & $36(85.7)$ \\
$11-20$ & $4(9.52)$ \\
$\geq 20$ & $2(4.76)$ \\
Total & $42(100)$ \\
\hline
\end{tabular}

Table 2: Distribution of medulloblastoma cases according to histologic types

\begin{tabular}{ll}
\hline Histologic types of medulloblastoma & Frequency (\%) \\
\hline Classic medulloblastoma & $31(73.80)$ \\
Desmoplastic/nodular medulloblastoma & $6(14.28)$ \\
Medulloblastoma with extensive nodularity & $3(7.14)$ \\
Anaplastic medulloblastoma & $2(4.76)$ \\
Total & $42(100)$ \\
\hline
\end{tabular}

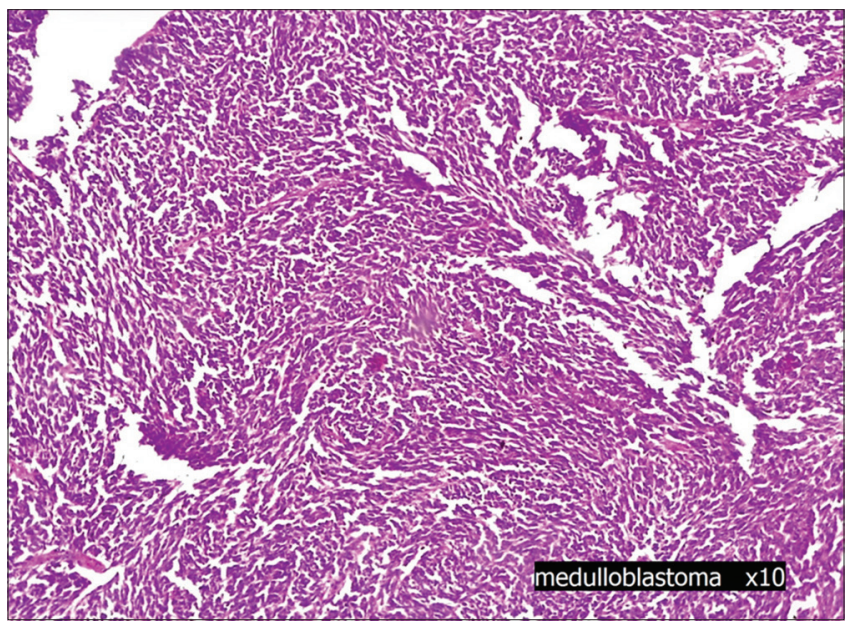

Fig. 1: $(\times 100)$ Hematoxylin and eosin stained histological section for 5-year-old child with a classic medulloblastoma demonstrates small round blue cells morphology 


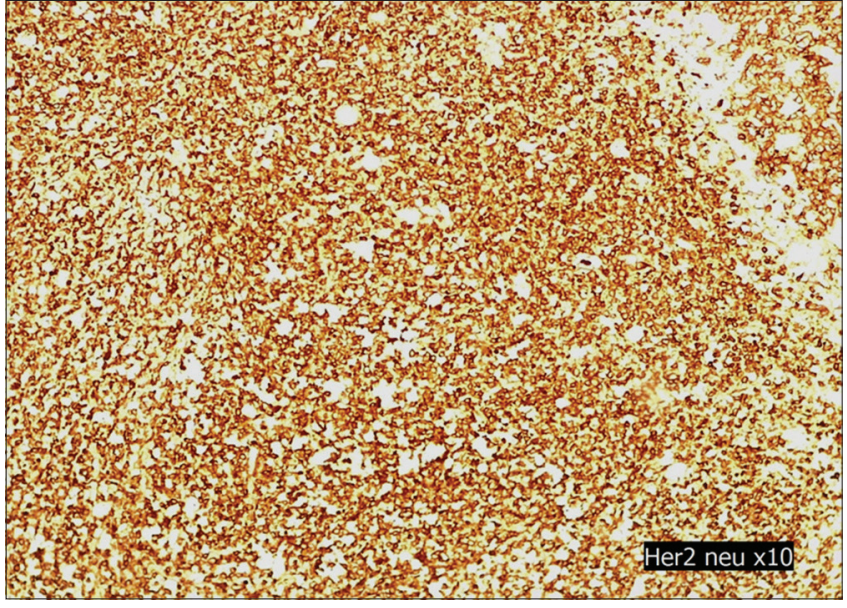

Fig. 2: $(\times 100)$ Immunohistochemically stained section of medulloblastoma with human epidermal growth factor receptor 2/NEU antibody demonstrates strong positive membranous staining in more than $10 \%$ of tumor cells

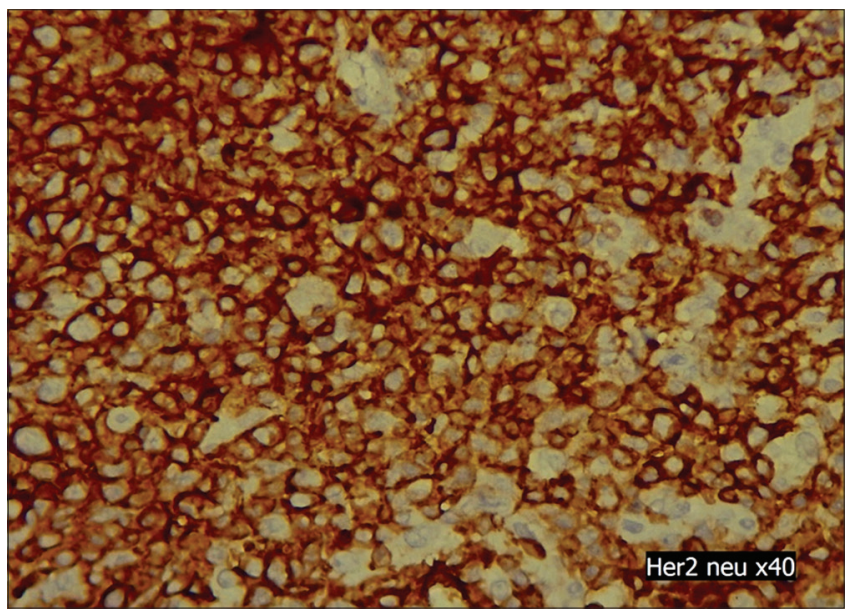

Fig. 3: (x 400) Immunohistochemically stained section of medulloblastoma with human epidermal growth factor receptor $2 / \mathrm{NEU}$ antibody demonstrates strong positive membranous staining in more than $10 \%$ of tumor cells

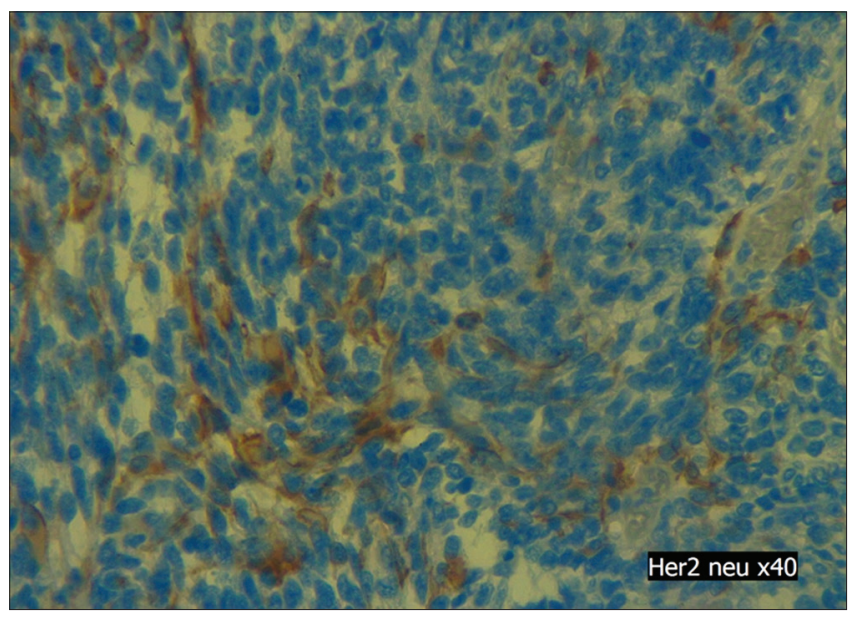

Fig. 4: $(\times 400)$ Immunohistochemical stained section of medulloblastoma with human epidermal growth factor receptor

2/NEU antibody demonstrates the negative result (only cytoplasmic staining pattern)

There are fewstudiesworldwide focused on the overexpression of HER2/ NEU in medulloblastoma, the present study is the first study in Iraq searching for this purpose in an attempt not only to give an impression on the prognosis but also to help in identifying which patient would benefit from the application of the promising targeted therapy based on the ongoing clinical trials. The study revealed that only two cases (represent $4.76 \%$ ) showed positive strong membranous expression of this marker, this result is relatively low compared to previous similar studies worldwide [13,14,23,24]. However, there are some discrepancies in the reporting rate of HER2/NEU immunoreactivity in the literature and most of the reference texts due to the morphological characteristic of medulloblastoma cells, which have a scarce cytoplasm, making a difficulty in ascribing the immunohistochemical staining whither to the membrane or to the cytoplasm [13]. To improve a better quality of results, the process of staining of all samples was done in two different sites, in governmental hospital using an autostainer and in the private lab bimanually, positive and negative controls were included in each run. A number of reasons should be discussed that hopefully explain a low expression rate of HER2/NEU obtained in this study. Despite developing ideas and clinical researches about the probable use of anti-HER2/NEU drugs in medulloblastoma; unfortunately,there is no well-established standardized criteria currently available for the immunohistochemical methods (ie, tissue handling/fixation, antibodies used, controls, and artifacts) and for interpretation and scoring of HER2 immunohistochemical results in medulloblastoma util now unlike in breast cancer and this issue is problematic because the most important factor predict patient response to targeted therapy is an accurate and reliable searching test. On the other hand, this study used the same scoring system established for breast cancer and it probably may not be suitable for other cancer with HER2/NEU expression, this fact approved by a recent large international clinical trial on the use of targeted antiHER2/NEU therapy in gastric cancer (Trastuzumab for Gastric Cancer) in which the positivity (therefore the benefit from targeted therapy) was accepted as cytoplasmic and incomplete membranous staining patterns [25]. Accordingly, the interpretation guidelines in biologically and pathogenetically different tumor types could not be simply applied because this result in unreliable immunohistochemical interpretation so the specific scoring system should be urgently established. To reach this purpose, it's important to illustrate the pattern of immunohistochemical staining in both positive and negative cases, in this study 14/42 (33.3\%) showed incomplete membranous staining and 5/42 (11.9\%) showed only cytoplasmic reactivity. Although cytoplasmic or incomplete membranous staining has been considered unspecific or art factual, therefore according to the above explanation they should be investigated for targeted therapy response. Although this new era brings promise, the limited numbers of medulloblastoma cases often remain a major work impediments.

Furthermore, the complex issue of intratumoral heterogeneity regarding HER2/NEU over expression and amplification which is already present in a subset of breast cancer cases both at inter and intratumoral level is regarded by most authors as an unavoidable factor for accurate HER2 status assessment [26,27]. From this point of view, low expression rates in this study may reflect a high intratumoral heterogeneity for HER2/ NEU expression in medulloblastoma which if it is confirmed will open a challenging field of research in future.

Gilbertson et al. had demonstrated in a retrospective study of 55 cases of MB, the prognostic value of HER2/NEU expression was dependent on the percentage of positive tumor cells $[13,14]$, these observations necessitate the development of medulloblastoma - specific HER2 histological scoring criteria and immunohistochemical testing methods. Because this is the first Iraqi study, as a starting point we thought we should stick to the scoring criteria of the original HER2/NEU scoring in breast cancer (established by more than 10 years experience in HER2/ NEU overexpression study and clinical correlation with treatment response) aspiring that it becomes a basic template for more future studies about the overexpression of HER2/NEU in medulloblastoma in conjunction with other ancillary tests and correlate them with clinical response to targeted therapy to identify the unique features of HER2/NEU overexpression in medulloblastoma with the final goal 
of establishing medulloblastoma - specific HER2 histological scoring criteria and immunohistochemical testing methods to standardize immunohistochemistry for this application.

\section{CONCLUSION}

The rate of expression of HER2/NEU in medulloblastoma among Iraqi children is very low and found only in aggressive anaplastic histological type when immunohistochemically evaluated using the same scoring system established for HER2/NEU in breast cancer. However, a good number of negative cases showed cytoplasmic and incomplete membranous staining patterns highlighting the importance of establishing medulloblastoma - specific HER2 scoring criteria and testing methods to discover the unique feature of expression of this therapeutic target in medulloblastoma.

\section{AUTHOR'S CONTRIBUTION}

First author: Study Design, diagnosis, data analysis, and editing the manuscript.

Second author: Conducted laboratory tests, diagnosis.

Third author: Diagnosis, data analysis, and editing the manuscript.

All authors agreed to be accountable for all aspects of the work ensuring the accuracy and integrity of any part of the work.

\section{CONFLICTS OF INTEREST}

The authors declare that we do not have any conflicts of interest.

\section{REFERENCES}

1. Roussel MF, Hatten ME. Cerebellum development and medulloblastoma. Curr Top Dev Biol 2011:94:235-82.

2. Kumar V, Kumar V, McGuire T, Coulter DW, Sharp JG, Mahato RI, et al. Challenges and recent advances in medulloblastoma therapy. Trends Pharmacol Sci 2017;38:1061-84.

3. Gilbertson RJ. Medulloblastoma: Signalling a change in treatment. Lancet Oncol 2004;5:209-18.

4. Northcott PA, Jones DT, Kool M, Robinson GW, Gilbertson RJ, Cho YJ, et al. Medulloblastomics: The end of the beginning. Nat Rev Cancer 2012:12:818-34.

5. Kijima N, Kanemura Y. Molecular classification of medulloblastoma. Neurol Med Chir (Tokyo) 2016:56:687-97.

6. Massimino M, Antonelli M, Gandola L, Miceli R, Pollo B, Biassoni V, et al. Histological variants of medulloblastoma are the most powerful clinical prognostic indicators. Pediatr Blood Cancer 2013;60:210-6.

7. Kool M, Korshunov A, Remke M, Jones DT, Schlanstein M, Northcott PA, et al. Molecular subgroups of medulloblastoma: An international meta-analysis of transcriptome, genetic aberrations, and clinical data of WNT, SHH, group 3, and group 4 medulloblastomas. Acta Neuropathol 2012;123:473-84.

8. Silva Rd, Marie SK, Uno M, Matushita H, Wakamatsu A, Rosemberg S, et al. CTNNB1, AXIN1 and APC expression analysis of different medulloblastoma variants. Clinics (Sao Paulo) 2013;68:167-72

9. Taylor MD, Northcott PA, Korshunov A, Remke M, Cho YJ, Clifford SC, et al. Molecular subgroups of medulloblastoma: The current consensus. Acta Neuropathol 2012;123:465-72.

10. Vinod K, Virender K, Timothy M, Donald W, Coulter JG, Ram IM. challenges and recent advances in medulloblastoma. Ther Trends Pharm Sci 2013;8:1061-84.

11. Buza N, Roque DM, Santin AD. HER2/neu in endometrial cancer: A Promising therapeutic target with diagnostic challenges. Arch Pathol Lab Med 2014;138:343-50.

12. Perez EA, Romond EH, Suman VJ, Jeong JH, Sledge G, Geyer CE Jr, et al. Trastuzumab plus adjuvant chemotherapy for human epidermal growth factor receptor 2-positive breast cancer: Planned joint analysis of overall survival from NSABP B-31 and NCCTG N9831. J Clin Oncol 2014;32:3744-52.

13. Gilbertson RJ, Pearson AD, Perry RH, Jaros E, Kelly PJ. Prognostic significance of the c-erbB-2 oncogene product in childhood medulloblastoma. Br J Cancer 1995;71:473-7.

14. Gilbertson RJ. ERBB2 in pediatric cancer: Innocent until proven guilty. Oncologist 2005;10:508-17.

15. Holla SN, Nayak V, Bairy KL, Tripathy A, Holla S. Her-2 gene, receptors and drug target: A systematic review. Int J Pharm Pharm Sci 2016;8:4-9.

16. Ahmed N, Ratnayake M, Savoldo B, Perlaky L, Dotti G, Wels WS, et al. Regression of experimental medulloblastoma following transfer of HER2-specific T cells. Cancer Res 2007;67:5957-64.

17. Gupta M, Dahiya J, Marwaha RK, Dureja H. Therapies in cancer treatment: AN overview. Int J Pharm Pharm Sci 2015;4:1-9.

18. Mates M, Fletcher GG, Freedman OC, Eisen A, Gandhi S, Trudeau ME, et al. Systemic targeted therapy for her2-positive early female breast cancer: A systematic review of the evidence for the 2014 cancer care ontario systemic therapy guideline. Curr Oncol 2015;22:S114-22.

19. Chan A, Delaloge S, Holmes FA, Moy B, Iwata H, Harvey VJ, et al. Neratinib after trastuzumab-based adjuvant therapy in patients with HER2-positive breast cancer (ExteNET): A multicentre, randomised, double-blind, placebo-controlled, phase 3 trial. Lancet Oncol 2016;17:367-77.

20. Kumar LP, Deepa SF, Moinca I, Suresh P, Naidu KV. Medulloblastoma: A common pediatric tumor: Prognostic factors and predictors of outcome. Asian J Neurosurg 2015;10:50.

21. Curran EK, Sainani KL, Le GM, Propp JM, Fisher PG. Gender affects survival for medulloblastoma only in older children and adults: A study from the surveillance epidemiology and end results registry. Pediatr Blood Cancer 2009;52:60-4.

22. Herms JW, Behnke J, Bergmann M, Christen HJ, Kolb R, Wilkening $\mathrm{M}$, et al. Potential prognostic value of C-erbB-2 expression in medulloblastomas in very young children. J Pediatr Hematol Oncol 1997; 19:510-5.

23. Nam DH, Wang KC, Kim YM, Chi JG, Kim SK, Cho BK. The effect of chromosome $17 \mathrm{q}$ presence, proliferative and apoptotic indices, expression of C-erbB-2, bcl-2 and p53 proteins on the prognosis of medulloblastoma. J Korean Med Sci 2000;15:452-6.

24. Meurer RT, Martins DT, Hilbig A, Ribeiro Mde C, Roehe AV, BarbosaCoutinho LM, et al. Immunohistochemical expression of markers ki67, neun, synaptophysin, p53 and HER2 in medulloblastoma and its correlation with clinicopathological parameters. Arq Neuropsiquiatr 2008;66:385-90

25. Hofmann M, Stoss O, Shi D, Büttner R, van de Vijver M, Kim W, et al. Assessment of a HER2 scoring system for gastric cancer: Results from a validation study. Histopathology 2008;52:797-805.

26. Perez EA, Press MF, Dueck AC, Jenkins RB, Kim C, Chen B, et al. Immunohistochemistry and fluorescence in situ hybridization assessment of HER2 in clinical trials of adjuvant therapy for breast cancer (NCCTG N9831, BCIRG 006, and BCIRG 005). Breast Cancer Res Treat 2013;138:99-108.

27. Cottu PH, Asselah J, Lae M, Pierga JY, Diéras V, Mignot L, et al. Intratumoral heterogeneity of HER2/neu expression and its consequences for the management of advanced breast cancer. Ann Oncol 2008;19:595-7. 